\title{
Bearing and Range Estimation Algorithm for Buried Object in Underwater Acoustics
}

\author{
Dong Han, Caroline Fossati, Salah Bourennane, and Zineb Saidi \\ Institut Fresnel (UMR CNRS 6133), Faculté des Sciences et Techniques de Saint Jérôme, 13397 Marseille cedex 20, France
}

Correspondence should be addressed to Salah Bourennane, salah.bourennane@fresnel.fr

Received 28 June 2009; Accepted 19 July 2009

Recommended by Miguel Lagunas

\begin{abstract}
A new algorithm which associates (Multiple Signal Classification) MUSIC with acoustic scattering model for bearing and range estimation is proposed. This algorithm takes into account the reflection and the refraction of wave in the interface of watersediment in underwater acoustics. A new directional vector, which contains the Direction-Of-Arrival (DOA) of objects and objectssensors distances, is used in MUSIC algorithm instead of classical model. The influence of the depth of buried objects is discussed. Finally, the numerical results are given in the case of buried cylindrical shells.
\end{abstract}

Copyright ( 12009 Dong Han et al. This is an open access article distributed under the Creative Commons Attribution License, which permits unrestricted use, distribution, and reproduction in any medium, provided the original work is properly cited.

\section{Introduction}

The main target of Array processing is to estimate the bearing and range of sources or objects radiating in a medium of propagation [1]. MUSIC is one of the commonly used high resolution algorithms for DOA estimation. It uses the orthogonality property between the signal subspace and the noise subspace to localize sources [2].

In this letter, we propose a new algorithm for bearing and range estimation of buried objects in underwater acoustics. The approach is based on MUSIC combined with the acoustic scattering model $[3,4]$. We consider the reflection and the refraction of wave at water-sediment interface. This method develops a new source steering vector including the information of bearing and range of buried objects. The vector is used in MUSIC algorithm instead of the classical plane wave model [5]. The attenuation in the sediment is distinct for the objects buried deep, so we discuss the influence of the depth of buried objects. The proposed algorithm is evaluated by numerical simulations in the case of buried cylindrical shells.

The remainder of the letter is organized as follows. Section 2 summarizes the problem formulation. In Section 3, the scattering acoustic model of generating the received signals is discussed. An algorithm is proposed in Section 4. Next, the influence of the depth of buried objects is presented in Section 5. Finally some numerical results are addressed in Sections 5 and 6 conclude the paper.

Throughout the paper, lowercase boldface letters represent vectors, uppercase boldface letters represent matrices, and lower and uppercase letters represent scalars. The symbol " $T$ " is used for transpose operation. The superscript " + " is used to denote complex conjugate transpose and $\|\cdot\|$ denotes the $L_{2}$ norm for complex vectors.

\section{Problem Formulation}

Consider a linear array of $N$ sensors receives the signals scattered from $K$ objects $(K<N)$. The received signal can be grouped into a vector $\mathbf{x}\left(f_{l}\right)$ written as

$$
\mathbf{x}\left(f_{l}\right)=\mathbf{A}\left(f_{l}\right) \mathbf{s}\left(f_{l}\right)+\mathbf{b}\left(f_{l}\right),
$$

where $\mathbf{A}\left(f_{l}\right)$ is the transfer matrix, $\mathbf{s}\left(f_{l}\right)$ is the vector of signal, and $\mathbf{b}\left(f_{l}\right)$ is the vector of additive Gaussian noise.

The wavefront is assumed to be plane when the objects are far from the array. We use MUSIC algorithm to estimate the angle $\theta$ of the plane wave associated with the objects

$$
\operatorname{MUSIC}(\theta)=\frac{1}{\mathbf{a}(\theta)^{+} \mathbf{V}_{\mathbf{b}} \mathbf{V}_{\mathbf{b}}{ }^{+} \mathbf{a}(\theta)},
$$

where $\mathbf{a}(\theta)=\left[1, e^{-j \varphi}, \ldots, e^{-(N-1) j \varphi}\right]^{T}$ is the steering vector and $\varphi=2 \pi f(d \sin (\theta) / c), \mathbf{V}_{\mathbf{b}}$ is the the matrix of eigenvectors 


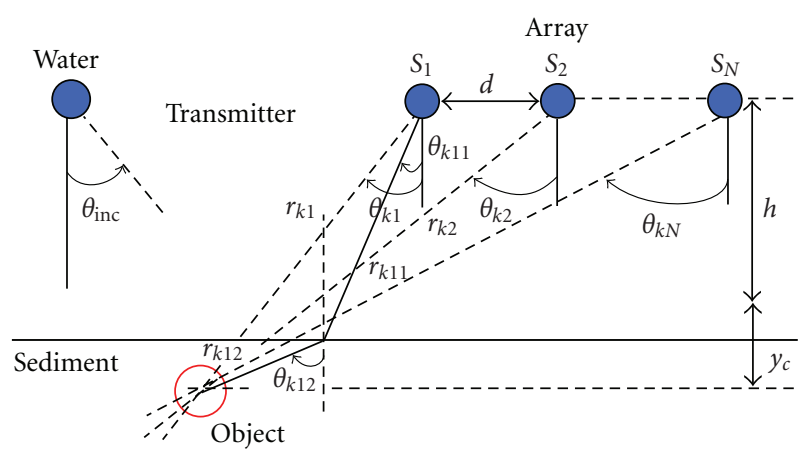

FIGURE 1: Geometry configuration of the buried object.

spanned by the noise subspace, $c$ is the sound speed, $d$ is the distance of sensors, and $j$ is the complex operator.

\section{Scattering Acoustic Model: To Generate the Received Signals}

We assume an object is buried in the sediment $\left(\theta_{k 1}, r_{k 1}\right)$ associated to the first sensor of the array. An incident plane wave propagating in the water reaches the interface with $\theta_{\text {inc }}$ (see Figure 1). A reflected plane wave is generated in the water and a refracted plane wave is propagated in the sediment. So the array receives three components [6]:

(i) the incident plane wave,

(ii) the reflecting plane wave,

(iii) the transmitted plane wave diffused by the object.

The pressures in the water and the sediment are given by five unknown parameters $\theta_{k 11}, r_{k 11}, \theta_{k 12}, r_{k 12}$, and the depth of buried object $y_{c}$ based on $\theta_{k 1}$ and $r_{k 1}$ (see Figure 1):

$$
\begin{gathered}
y_{c}=r_{k 1} \cos \left(\theta_{k 1}\right)-h, \\
\theta_{k 12}=\arcsin \left(\frac{c_{2}}{c_{1}} \sin \left(\theta_{\text {inc }}\right)\right), \\
r_{k 12}=\frac{r_{k 1} \cos \left(\theta_{k 1}\right)-h}{\cos \left[\arcsin \left(\left(c_{2} / c_{1}\right) \sin \left(\theta_{\text {inc }}\right)\right)\right]}, \\
\theta_{k 11}=\arctan \left[\frac{r_{k 1} \cos \left(\theta_{k 1}\right)-r_{k 12} \cos \left(\theta_{k 12}\right)}{r_{k 1} \sin \left(\theta_{k 1}\right)-r_{k 12} \sin \left(\theta_{k 12}\right)}\right], \\
r_{k 11}=\frac{h}{\cos [\arctan (Q)]},
\end{gathered}
$$

where $Q$ denotes $\left(r_{k 1} \cos \left(\theta_{k 1}\right)-r_{k 12} \cos \left(\theta_{k 12}\right)\right) /\left(r_{k 1} \sin \left(\theta_{k 1}\right)-\right.$ $\left.r_{k 12} \sin \left(\theta_{k 12}\right)\right)$

We consider the case of infinitely long elastic cylinder shell. The first sensor of the array $P_{c y l}^{\text {tot }}$ receives the acoustic pressure components as follows:

$$
\begin{aligned}
P_{\text {cyl }}^{\text {tot }}\left(\theta_{k 1}, r_{k 1}\right)= & P_{\text {inwater }}\left(\theta_{k 1}, r_{k 1}\right)+P_{\text {refwater }}\left(\theta_{k 1}, r_{k 1}\right) \\
& +P_{\text {diffcyl }}\left(\theta_{k 1}, r_{k 1}\right),
\end{aligned}
$$

where $P_{\text {inwater }}$ is the pressure incident in the water:

$$
P_{\text {inwater }}\left(\theta_{k 1}, r_{k 1}\right)=e^{j k_{1}\left(-\left(r_{k 1} \sin \left(\theta_{k 1}\right)\right) \sin \left(\theta_{\text {inc }}\right)+h \cos \left(\theta_{\text {inc }}\right)\right)}
$$

$P_{\text {refwater }}$ is the pressure reflected by the sediment-water interface:

$$
P_{\text {refwater }}\left(\theta_{k 1}, r_{k 1}\right)=R e^{j k_{1}\left(\left(r_{k 1} \sin \left(\theta_{k 1}\right)\right) \sin \left(\theta_{\text {inc }}\right)-h \cos \left(\theta_{\text {inc }}\right)\right)},
$$

where $R$ is the reflection coefficient of the interface.

$P_{\text {diffcyl }}^{t}\left(\theta_{k 1}, r_{k 1}\right)$ is the diffused acoustic pressure wave transmitted in the water [7]:

$$
P_{\text {diffcyl }}^{t}\left(\theta_{k 1}, r_{k 1}\right)=\sum_{m=-\infty}^{+\infty} \xi \mathbf{T}_{c}\left(\mathbf{I}-\mathbf{D}_{c}\right)^{-1} \psi_{\text {cyl }}^{t},
$$

where $\mathbf{I}$ is the identity matrix, $\mathbf{D}_{c}$ is a linear operator, $\mathbf{T}_{c}$ is the transition diagonal matrix, $\psi_{\text {cyl }}^{t}$ is the vector of transmitted wave, and $\xi=\left[\xi_{1}, \xi_{2}, \ldots, \xi_{m}\right]$ is defined by

$$
\xi_{m}=T_{\text {water-sed }}\left(\theta_{\text {inc }}\right) e^{j k_{2} y_{c} \cos \left(\theta_{k 11}\right)} j^{m} e^{-j m\left(\pi-\theta_{k 11}\right)},
$$

where $T_{\text {water-sed }}$ is the transmission coefficient.

\section{Algorithm for Bearing and Range Estimation of Buried Objects}

(1) Find an initial estimation of $\theta, r$, and the number of objects $K$ by the beam forming method.

(2) Fill the matrice $\mathbf{A}\left(f_{l}\right)=\left[\mathbf{a}\left(f_{l}, \theta_{1}, r_{1}\right), \ldots, \mathbf{a}\left(f_{l}, \theta_{k}, r_{k}\right)\right]$ and the components are filled with cylindrical scattering model $\mathbf{a}\left(f_{l}, \theta_{k}, r_{k}\right)=$ $\left[P_{\text {cyl }}^{\text {tot }}\left(f_{l}, \theta_{k 1}, r_{k 1}\right), \ldots, P_{\text {cyl }}^{\text {tot }}\left(f_{l}, \theta_{k N}, r_{k N}\right)\right]^{T} \quad$ for $k=1,2, \ldots, K$. The first vector is given by (4). The other $P_{\text {cyl }}^{\text {tot }}\left(f_{l}, \theta_{k i}, r_{k i}\right)$ for $i=2, \ldots, N$ associated with the $i$ th sensor can be formed by (see Figure 1)

$$
\begin{aligned}
& r_{k i}=\sqrt{r_{k i-1}^{2}+d^{2}-2 r_{k i-1} d \cos \left(\frac{\pi}{2}+\theta_{k i-1}\right)}, \\
& \theta_{k i}=-\frac{\pi}{2}+\cos ^{-1}\left(\frac{d^{2}+r_{k i}^{2}-r_{k i-1}^{2}}{2 r_{k i-1} d}\right), \quad i=2, \ldots, N .
\end{aligned}
$$

(3) Estimate the spectral matrix $\boldsymbol{\Gamma}\left(f_{l}\right)=E\left[\mathbf{x}\left(f_{l}\right) \mathbf{x}^{+}\left(f_{l}\right)\right]$.

(4) Calculate sources spectral matrices by

$$
\begin{aligned}
\boldsymbol{\Gamma}_{\mathbf{s}}\left(f_{l}\right)= & \left(\mathbf{A}^{+}\left(f_{l}\right) \mathbf{A}\left(f_{l}\right)\right)^{-1} \mathbf{A}^{+}\left(f_{l}\right) \\
& \times\left[\boldsymbol{\Gamma}\left(f_{l}\right)-\hat{\sigma}^{2}\left(f_{l}\right) \mathbf{I}\right] \mathbf{A}\left(f_{l}\right)\left(\mathbf{A}^{+}\left(f_{l}\right) \mathbf{A}\left(f_{l}\right)\right)^{-1}
\end{aligned}
$$

where $\mathbf{I}$ is the identity matrix and $\hat{\sigma}^{2}$ is the noise variance.

(5) Compute the average of the spectral matrices

$$
\overline{\boldsymbol{\Gamma}}_{s}\left(f_{0}\right)=\frac{1}{L} \sum_{l=1}^{L} \boldsymbol{\Gamma}_{\mathbf{s}}\left(f_{l}\right), \quad 1 \leq l \leq L,
$$

where $L$ represents the number of frequencies and $f_{0}$ is the center frequency of the spectrum of the received signals.

(6) Caltulate $\overline{\boldsymbol{\Gamma}}\left(f_{0}\right)=\mathbf{A}\left(f_{0}\right) \overline{\boldsymbol{\Gamma}}_{\mathbf{s}}\left(f_{0}\right) \mathbf{A}^{+}\left(f_{0}\right)$. 


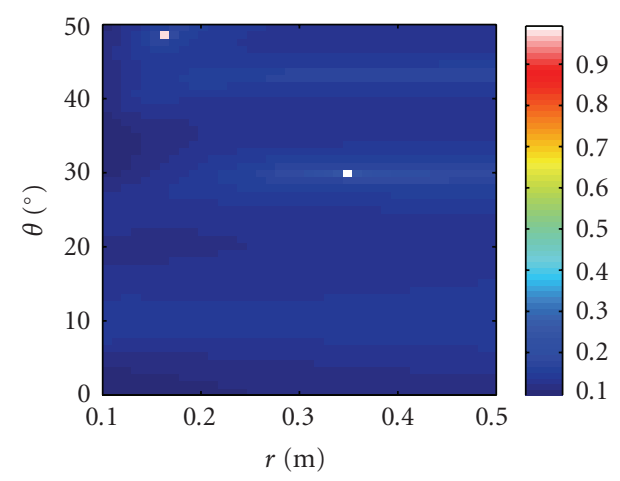

FIGURE 2: Localization of cylindrical shells.

(7) Use the eigenvectors $\mathbf{T}\left(f_{0}, f_{l}\right)=\mathbf{V}\left(f_{0}\right) \mathbf{V}^{+}\left(f_{l}\right)$ to obtain the focusing operator, where $\mathbf{V}\left(f_{0}\right)$ and $\mathbf{V}\left(f_{l}\right)$ are, respectively, the eigenvector matrices of $\overline{\boldsymbol{\Gamma}}\left(f_{0}\right)$ and $\boldsymbol{\Gamma}\left(f_{l}\right)$.

(8) Calculate the focused spectral matrix:

$$
\widehat{\boldsymbol{\Gamma}}\left(f_{0}\right)=\frac{1}{L} \sum_{l=1}^{L} \mathbf{T}\left(f_{0}, f_{l}\right) \boldsymbol{\Gamma}\left(f_{l}\right) \mathbf{T}^{+}\left(f_{0}, f_{l}\right) .
$$

(9) Estimate the number of objects by AIC or MDL [8].

Calculate the spatial spectrum of MUSIC method for estimating bearing and range of buried objects:

$$
\operatorname{MUSIC}\left(f_{0}, \theta_{k}, r_{k}\right)=\frac{1}{\left\|\mathbf{a}\left(f_{0}, \theta_{k}, r_{k}\right)^{+} \mathbf{V}_{\mathbf{b}}\left(f_{0}\right)\right\|},
$$

where $\mathbf{V}_{\mathbf{b}}\left(f_{0}\right)$ is the eigenvector matrix of noise subspace.

\section{Influence of the Depth of Buried Objects}

In sandy sediment, the attenuating effect of suspended material is negligible. Conversely, the attenuating effect of the sediment is significant. It can be reported in $\mathrm{dB} / \mathrm{cm} / \mathrm{kHz}$ since the examination of attenuation yielded a linear dependency with frequency. The attenuation coefficient of common sand [9] is $\alpha_{\text {att }}=0.5 \mathrm{~dB} / \mathrm{cm} / \mathrm{kHz}$.

\section{Numerical Results}

The parameters of the simulations are defined as follows: $d$ is $0.002 \mathrm{~m}, N$ is 10 , the frequency band is $[200,300] \mathrm{kHz}$, and the signal frequency $f$ is $250 \mathrm{kHz}$. The wave speed in the water $c_{1}$ is $1500 \mathrm{~m} / \mathrm{s}$ and in the sediment $c_{2}$ is $1700 \mathrm{~m} / \mathrm{s}$. The water density $D_{1}$ is $1000 \mathrm{~kg} / \mathrm{m}^{3}$, the sediment density $D_{2}$ is $1500 \mathrm{~kg} / \mathrm{m}^{3}$. The incidence angle $\theta_{\text {inc }}$ is $60^{\circ}$.

The array is placed in the water with the hight $h=$ $0.1 \mathrm{~m}$. The variance of the noise $\sigma^{2}$ is 100 and SNR is $30 \mathrm{~dB}$. As shown in Figure 2, the white points coordinate two cylindrical shells $\left(30^{\circ}, 0.35 \mathrm{~m}\right)$ and $\left(49^{\circ}, 0.16 \mathrm{~m}\right)$.

When the cylindrical shell is deeply buried $\left(20^{\circ}, 0.3 \mathrm{~m}\right)$, we vary the interface of water-sediment for each $y_{c}$. The

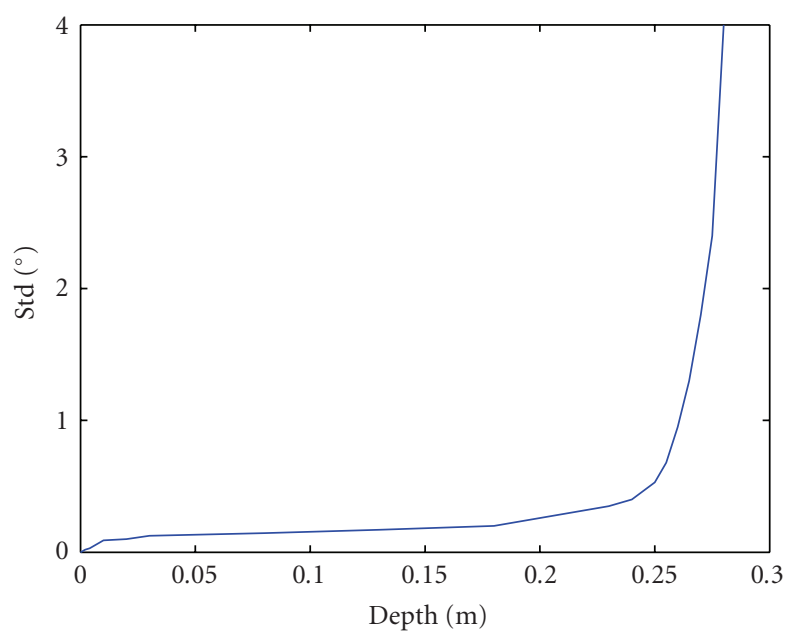

FIGURE 3: Std between expected and estimated bearings.

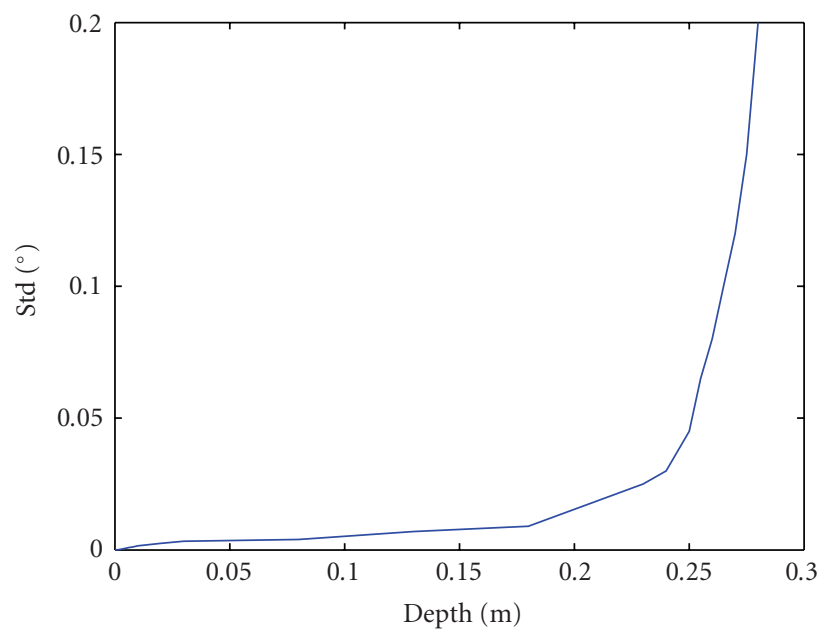

Figure 4: Std between expected and estimated ranges.

signal may be unworkable if the object is buried deep. Furthermore, we evaluate statistically the influence of the depth of buried objects by Standard Deviation:

$$
\operatorname{Std}=\sqrt{\frac{1}{K} \sum_{k=1}^{K}\left[\left(X_{\text {exp }}-X_{\text {est }}\right)_{k}^{2}\right]},
$$

where $X$ is the bearing $\theta$ or the range $r$ and $K=100$.

The results obtained (see Figures 3 and 4 ) show that the algorithm is not efficient when the object is buried deeper than $0.25 \mathrm{~m}$.

\section{Conclusion}

In this paper, we propose a new algorithm based on MUSIC associated with acoustic scattering model for bearing and range estimation of buried objects. There is an analogy of the water-sediment interface by combining with the reflection and the refraction of wave in the model. A new directional vector, including the information for bearing and range 
estimation, is employed instead of the plane wave model in the MUSIC algorithm. The results of buried cylindrical shells are significantly accurate. Then we study the influence of the depth. The results show that beyond a certain depth, the attenuation becomes too large and therefore the objects cannot be detected or located neither.

\section{References}

[1] H. Krim and M. Viberg, "Two decades of array signal processing research: the parametric approach," IEEE Signal Processing Magazine, vol. 13, no. 4, pp. 67-94, 1996.

[2] R. Kumareasan and D. W. Tufts, "Estimating the angles of arrival of multiple source plane waves," IEEE Transactions on Aerospace and Electronic Systems, vol. 19, pp. 134-139, 1983.

[3] J. A. Fawcett, "Amethod of images for a penetrable acoustic waveguide," Journal of the Acoustical Society of America, vol. 113, no. 1, pp. 194-204, 2003.

[4] J. A. Fawcett and R. Lim, "Evaluation of the integrals of target/seabed scattering using the method of complex images," Journal of the Acoustical Society of America, vol. 114, no. 3, pp. 1406-1415, 2003.

[5] Z. Saidi and S. Bourennane, "Cumulant-based coherent signal subspace method for bearing and range estimation," EURASIP Journal on Advances in Signal Processing, vol. 2007, Article ID 84576, 2007.

[6] R. Lim, "Acoustic scattering by a partially buried threedimensional elastic obstacle," Journal of the Acoustical Society of America, vol. 104, no. 2, part 1, pp. 769-782, 1998.

[7] A. Coatanhay and J. M. Conoir, "Scattering by an elastic cylinder buried in a sedimentary fluidmedia," Journal of the Acoustical Society of America, vol. 103, no. 5, p. 2806, 1998.

[8] M. Wax and T. Kailath, "Detection of signals information theoretic criteria," IEEE Transaction on Acoustics, Speech and Signal Processing, vol. 33, no. 2, pp. 387-392, 1985.

[9] M. L. Oelze, W. D. O’Brien Jr., and R. G. Darmody, "Measurement of attenuation and speed of sound in soils," Soil Science Society of America Journal, vol. 66, no. 3, pp. 788-796, 2002. 

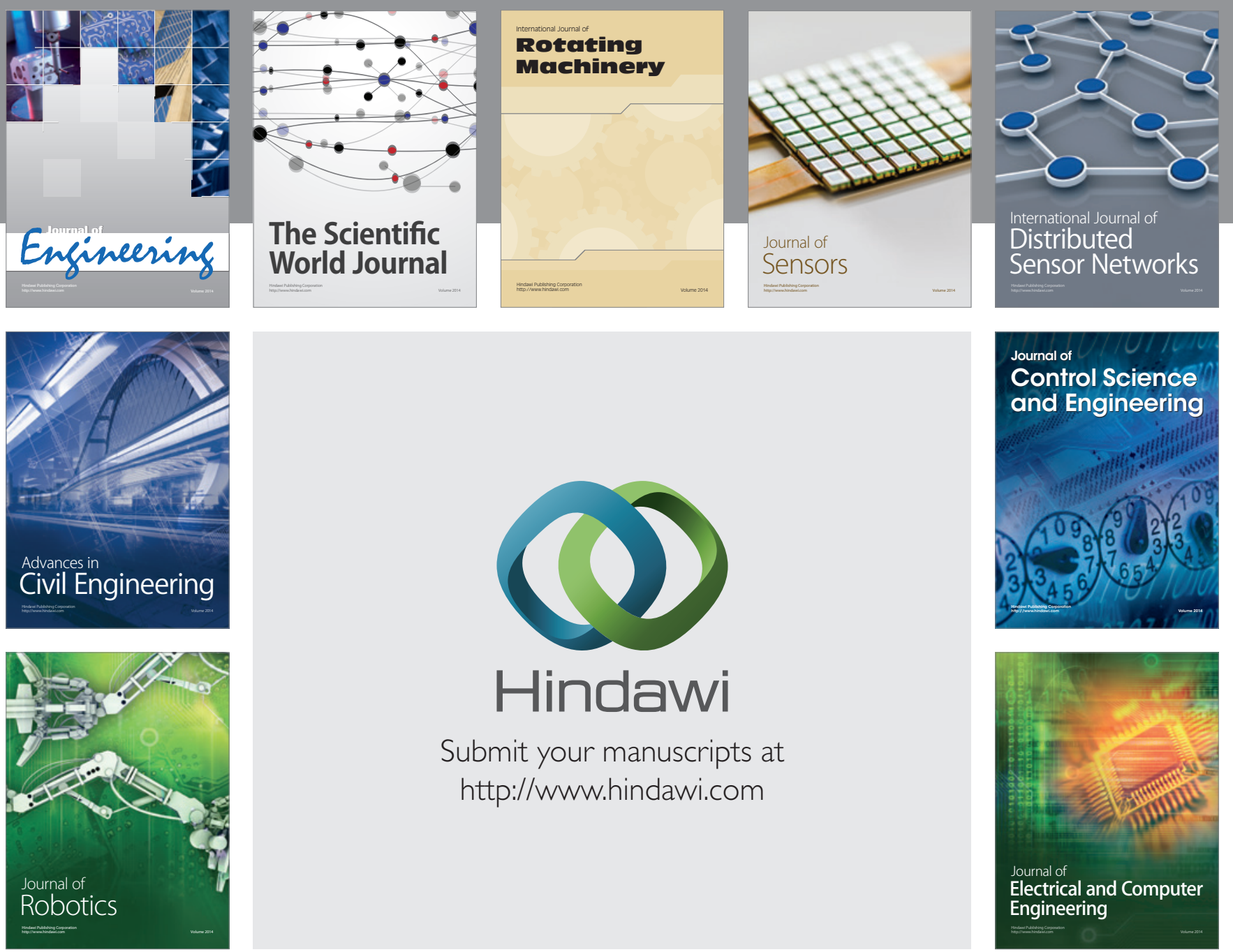

Submit your manuscripts at

http://www.hindawi.com
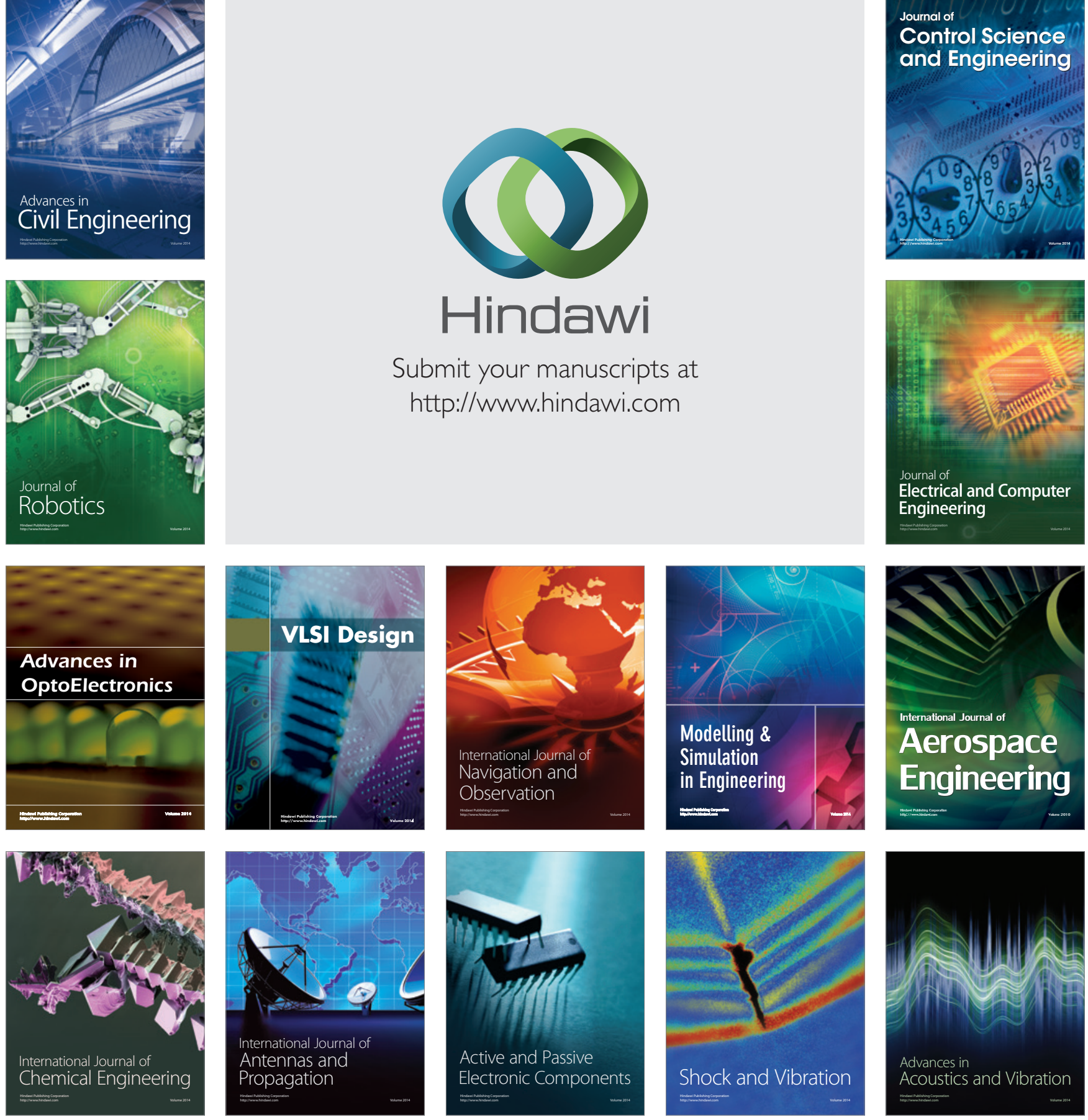